\title{
El impacto de Heidegger y Gadamer en la hermenéutica trascendental de Apel
}

\author{
The impact of Heidegger and Gadamer \\ on Apel's transcendental hermeneutics
}

GONZALO SCIVOLETTO

Conicet, Argentina

Recibido: $2 / \mathrm{X} / 2013 \quad$ Aceptado: 21/03/14

RESUMEN

El presente trabajo tiene por objetivo reconstruir sistemáticamente la «hermenéutica trascendental» de Karl-Otto Apel. En primer lugar, se describe el desarrollo de la interpretación apeliana de Heidegger, la cual consideramos que puede ser dividida en cuatro momentos. En segundo lugar, se explican los principales puntos de disenso de Apel con la hermenéutica filosófica de Gadamer. A lo largo del trabajo sugerimos, además, posibles caminos teóricos abiertos para la hermenéutica trascendental en tanto programa filosófico de investigación.

PALABRAS CLAVE

HERMENÉUTICA FILOSÓFICA, HERMENÉUTICA TRASCENDENTAL,

KARL-OTTO APEL

\begin{abstract}
This paper aims to systematically reconstruct «transcendental hermeneutics» of Karl-Otto Apel. Firstly, we describe the development of Heidegger's Apelian interpretation, which we consider can be divided into four stages. Secondly, we explain Apel's main points of disagreement with Gadamer's philosophical hermeneutics. Throughout the work we suggest further possible theoretical paths opened to transcendental hermeneutics as a philosophical research programme. KEY WORDS

PHILOSOPHIAL HERMENEUTICS, TRANSCENDENTAL HERMENEUTICS, KARL-OTTO APEL
\end{abstract}

(C) Contrastes. Revista Internacional de Filosofía, vol. XX-Nº1 (2015), pp. 47-66. ISSN: 1136-4076

Departamento de Filosofía, Universidad de Málaga, Facultad de Filosofía y Letras Campus de Teatinos, E-29071 Málaga (España) 


\section{INTRODUCCIÓN}

COMO GRAN PARTE de la problemática filosófica contemporánea, la obra de Karl-Otto Apel surge a partir de la filosofía de Heidegger y, especialmente, sus consecuencias ético-políticas. En el caso de Apel, el punto central de la confrontación con Heidegger gira en torno al concepto de verdad como aletheia y su respectiva subordinación a la temporalidad e historicidad (Apel 1998, p. $578)^{1}$. En efecto, la interpretación heideggeriana de la verdad como aletheia, esto es, en tanto acontecimiento, conduce, para Apel, a una oposición radical entre temporalidad (ser) y logos (razón) que destruye la posibilidad de la filosofía misma y de una moral universalista. Aún así, no es del todo sencillo reconstruir el proceso mediante el cual Apel llega a su posicionamiento respecto de Heidegger. Para ello es necesario conocer las interpretaciones que guían (o condicionan) la propia interpretación de Apel. Estas podrían clasificarse de la siguiente manera: 1) la interpretación «epistemológica» bajo el contexto de la escuela de Dilthey, en especial Erich Rothacker y Theodor Litt²; 2) la interpretación crítica de Ernst Tugendhat sobre el concepto de verdad en Heidegger (Tugendhat 1967, pp. 281-405); y 3) la interpretación «post-analítica» de Cristina Lafont, a partir de las teorías de la referencia directa (Lafont 1997) ${ }^{3}$. Es en el marco de las dos primeras lecturas, y consecuentemente, de la publicación de Verdad y Método de Gadamer, que Apel acuña el término hermenéutica

1 Debería agregarse aquí también el papel que cumple Wittgenstein, en relación con sus consecuencias relativistas. Sin embargo, mientras que, para Apel, la hermenéutica filosófica conduce a un relativismo diacrónico, el pragmatismo y la filosofía de los juegos del lenguaje conducen a un relativismo sincrónico (Apel 1998, pp. 18-19). Por otra parte, desde un punto de vista cronológico, como se mostrará en este trabajo, es decisivamente la filosofía de Heidegger, y sus consecuencias, la que motiva el filosofar de Apel. La filosofía de Wittgenstein muestra sus consecuencias «relativistas», para Apel, a través de Peter Winch. Para un análisis comparado, actualizado, de ambas tradiciones y sus consecuencias, véase el artículo de Apel «Wittgenstein und Heidegger. Kritische Wiederholung und Ergänzung eines Vergleichs» (Apel 1998, pp. 461503). Para la crítica a la «monadología de las formas de vida» en la que incurriría Winch, véase Scivoletto $2011 \mathrm{~b}$.

2 Peter Koslowski (1997) realiza una excelente caracterización de las ciencias del espíritu en la versión de Rothacker y muestra las relaciones que tiene con la filosofía posmoderna, especialmente con Lyotard. Creo que esta tesis de Koslowski refuerza la interpretación apeliana de la filosofía actual como una crítica total a la razón, motivada especialmente (aunque no exclusivamente) por la filosofía del destino del ser de Heidegger, esto es, como un historicismo radical. Por su parte, para una crítica de la crítica de Theodor Litt a Heidegger de la que se vale Apel, véase Bertorello 2007.

3 Para una discusión completa de la interpretación de Lafont de Ser y Tiempo, véase el Simposio publicado en el número 45 de la revista Inquiry: An Interdisciplinary Journal of Philosophy (2002). Véase además, Scivoletto 2011 a. 
trascendental en oposición a la hermenéutica filosófica, la cual, desde el punto de vista de Apel, posee consecuencias prácticas relativistas y abandona la cuestión de la corrección y el progreso de la comprensión (Apel 1998, pp. 571-607). En tal sentido, ya en sus primeros trabajos, Apel entiende que es posible reconducir la hermenéutica a un proyecto de filosofía trascendental, que el propio Heidegger y Gadamer respectivamente abandonarían. Hacia fines de los años 1960 Apel busca ampliar el marco de una hermenéutica trascendental hacia una pragmática trascendental hasta que, finalmente, acuña la expresión semiótica trascendental, la cual abarcaría tanto la hermenéutica como la pragmática trascendental. Es decir que el programa de una hermenéutica trascendental ocuparía, a primera vista, un lugar acotado en la obra de Apel, esto es, se circunscribiría aproximadamente a su producción filosófica de los años 1960. Sin embargo, Apel continúa utilizando la expresión hermenéutica trascendental en trabajos muy posteriores e incluso con la publicación de $\mathrm{Au}$ seinandersetzungen (1998) retoma discusiones con Heidegger y Gadamer que precisamente habían quedado circunscriptas a aquellos trabajos de los años 1960, e incluso su tesis doctoral de 1950, como se verá más adelante. Con ello, lo que se intenta mostrar en este trabajo es que la hermenéutica trascendental no se reduce a un momento histórico en el desarrollo de la filosofía apeliana, sino que puede constituirse en un campo propio de investigación filosófica. En el espíritu de la filosofía apeliana las investigaciones histórico-filosóficas sólo tienen sentido como parte de una problemática, un «asunto» en discusión.

Ahora bien, no es objetivo de este artículo abordar la arquitectónica de la filosofía de Apel, y en tal sentido delinear las relaciones entre hermenéutica y pragmática trascendental en el marco de una semiótica trascendental (o en su dimensión práctica con la ética discursiva), sino reconstruir la génesis de la hermenéutica trascendental a partir de la recepción crítica de la obra de Heidegger y Gadamer e indicar los principales puntos de confrontación. Se trata de una cuestión que en investigaciones recientes ha suscitado un nuevo interés. Se pueden mencionar como ejemplo los trabajos de A. Crelier (2010), W. Petras (2011). Crelier considera que el modelo de fundamentación última se acerca más a una forma hermenéutica-trascendental y que esta, a su vez, consiste en una profundización y «corrección» de la hermenéutica de la facticidad heideggeriana (Crelier 2010, p. 219 ss.). Por su parte, Petras, siguiendo a Burckhardt (2000), muestra la interrelación entre hermenéutica y pragmática trascendental y su complementariedad en una semiótica trascendental. Finalmente, en un encuentro realizado a comienzos de 2011 en Alemania con los principales representantes de la pragmática trascendental («Workshop Transzendentale Sprachpragmatik (TSP 2.0)»), Matthias Kettner ha planteado nuevamente la necesidad de repensar la hermenéutica trascendental de Apel y su «giro» de los años 1960. Por lo tanto, dentro de la tradición de la filosofía trascendental 
de Apel hay un cierto interés en reconsiderar los aportes de la hermenéutica filosófica. Sin embargo, lamentablemente no puede decirse lo mismo de la situación inversa, es decir, dentro de la hermenéutica filosófica no parece haber una evaluación más o menos positiva acerca de los posibles aportes de la filosofía de Apel a esta tradición. Jean Grondin, por mencionar un caso, se ha referido a la búsqueda de una fundamentación última como motivada por la «represión de nuestra finitud» y cuestiona el uso de la reflexión y la apelación a la autocontradicción performativa (Grondin 2003, p. 178 ss.). En relación con el concepto de verdad, Grondin (con Heidegger y Gadamer) rechaza, por ejemplo, la idea misma de «validez general»: «No se puede negar que la búsqueda de una verdad general implica el peligro de ocultar la realidad del entender (Verstehen) y de orientarlo hacia un ideal de conocimiento que nunca alcanzará» (Grondin 1999, p. 158). No obstante, en tal interpretación subyace, desde nuestro punto de vista, una lectura superficial de la filosofía apeliana, y es por ello que tal vez un análisis reconstructivo más preciso de la interpretación de la filosofía de Heidegger y Gadamer pueda aportar a un intercambio más fecundo entre hermenéutica filosófica y hermenéutica trascendental.

\section{Apel Y HeidegGer}

En el prólogo a una de sus obras fundamentales, Auseinandersetzungen in Erprobung des transzendentalespragmatischen Ansatzes, Apel explica el significado y la importancia del término "Auseinandersetzungen». Este término sintetiza, desde nuestro punto de vista, la concepción de la filosofía que se encuentra a la base de la filosofía discursiva de Apel. La idea central es que la filosofía no es un monólogo sino un diálogo, o mejor, una discusión argumentativa. Se trata de una discusión con alguien acerca de algo en principio controversial. Por lo tanto, lo que importa en una discusión filosófica es la cosa misma y no derrotar a un adversario, ni tampoco es un «mero comprender» el punto de vista del otro (aunque esto sea un elemento necesario, no es suficiente con ello), ni tampoco es un «entretenimiento», como puede ser un diálogo de café. En la controversia filosófica se ponen a consideración argumentos, se ofrecen mejores o peores razones, que por el hecho de la confrontación misma permiten un descentramiento del yo, esto es, de la propia posición, y un distanciamiento respecto de la posición del otro (Apel 1998, p. 7). En este sentido, la discusión de Apel con la hermenéutica filosófica (Heidegger y Gadamer) no es un elemento accidental, sino que es constitutivo de su propia producción filosófica. En el caso de Heidegger, sostendremos a modo de hipótesis que la interpretación apeliana atraviesa cuatro momentos. El primero y el segundo, que abarca desde su tesis doctoral hasta mediados de los años 1960, podría decirse presentan a un Apel muy heideggeriano, aunque ya incorpore inquietudes o preguntas de tipo kantianas. El tercero correspondería a su etapa crítica, 
registrada por el propio Apel en La transformación de la filosofía. El cuarto, que es el que proponemos como hipótesis de lectura, se correspondería con un distanciamiento crítico aún mayor, producto de la discusión de las teorías del significado en la tradición (post) analítica, especialmente con las teorías de la referencia directa y la crítica de Lafont a Heidegger desde ese marco teórico ${ }^{4}$.

\section{I.1 Problema heidegGeriano, mirada Kantiana}

El encuentro de Apel con la filosofía de Heidegger se inicia ya en su época de estudiante, y con su tesis doctoral Dasein und Erkennen. Eine erkenntnistheoretische Interpretation der Philosophie Martin Heideggers, bajo la dirección de Erich Rothacker. En ese entonces, el trasfondo (Hintergrund) de la interpretación apeliana de Heidegger lo conformaba el «principio de la significatividad“"(Satz der Bedeutsamkeit) de su maestro (Apel 1998, p. 513). El principio reza así: «sólo lo que me afecta, lo que para mí es, es decir, significa «algo», lo que despierta mi interés, lo que toca mi ser, lo que me parece digno de atención, digno de recordación, y finalmente digno de ulteriores pasos de apropiación verbal y mental, digno y merecedor de saberse, sólo esto tiene entrada, pasando por encima de este primero y más elemental umbral, en mi mundo» (Funke 1959, p. 172). Es probable, además, que sea esta influencia la que llevara a Apel a realizar, contra la advertencia del propio Heidegger, una interpretación «epistemológica“ de Ser y tiempo y a mantener durante toda su obra un interés por la fundamentación de las ciencias culturales, en línea con el neokantismo, y particularmente con Dilthey.

La otra influencia decisiva para la lectura de Heidegger es la de Kant. Y de hecho, ya desde el título de la tesis se puede observar una orientación

4 Se me ha observado, por parte de los/as evaluadores/as de este artículo, que otorgo demasiada importancia a la obra de Lafont, cuando la crítica a Heidegger de la que parte Apel ya está en Tugendhat. Si bien esto es cierto, y de hecho en esta suerte de periodización de la «recepción» de Heidegger por Apel la obra de Tugendhat tiene su momento, lo que intento señalar es que hay un aspecto «novedoso» en Lafont, que consiste en que la crítica que realiza a Heidegger se lleva a cabo desde el marco teórico del realismo interno de Putnam. También Apel se ocupa durante los años 1980 con las teorías del significado, tanto para cuestionar el giro intencionalista de Searle como la teoría de la referencia causal o directa de Kripke o Putnam. Pero, en ese sentido, tal crítica conduce a Apel a matizar o incluso poner en cuestionamiento algunos aspectos de su interpretación de la hermenéutica filosófica en los años 1960, con lo cual, podría al menos problematizarse la tesis de que la recepción de la hermenéutica filosófica es simplemente un momento ganado para la pragmática trascendental que luego permanece inalterable. Por otra parte, el valor de la interpretación de Lafont sobre Heidegger lo otorga el propio Apel, cuando afirma que representa una «revolución fundamental en la interpretación habitual de Heidegger» (Apel, 1998: 578 n.6). 
kantiana que permanecerá a lo largo de toda la obra de Apel. Este interés consiste esencialmente en la necesidad de una reconstrucción o renovación de la filosofía trascendental, pero sin dejar de lado la historicidad y la finitud del comprender en la línea de Heidegger. De allí el nombre que luego diera Apel a su propuesta filosófica: «hermenéutica (Heidegger)-trascendental (Kant)». De este modo, Apel planea su tesis doctoral como un proyecto en sentido inverso al de Heidegger. En lugar de partir de Ser y Tiempo hacia una reconstrucción o «traducción» de la Crítica de la Razón Pura en términos de analítica existencial (como intenta llevar a cabo Heidegger en Kant y el problema de la metafísica), Apel partirá de la preocupación kantiana por las condiciones de posibilidad y validez del conocimiento. En Dasein und Erkennen esta tarea se despliega en tres momentos, los cuales mencionaremos sólo a título ilustrativo. El primer momento, que comprende los capítulos I IV, lleva por título «El planteamiento de la pregunta trascendental como clave para la existencialidad (Existenzialität) del Dasein». El segundo, capítulos IV a IX, se denomina «La hermenéutica existencial (existenziale Hermeneutik) del ser en el mundo que comprende como crítica de la razón finita (endliche Vernunft)». En la tercera parte, «Resumen y perspectiva», se recogen las conclusiones generales, divididas en dos tipos de contribuciones: la primera a la interpretación de Heidegger y la segunda a una «Teoría existencial del conocimiento». En cuanto al planteo global de esta obra y los elementos que se encuentran en continuidad con el desarrollo posterior de la filosofía apeliana, seguimos a Boccignone (2004) y Mendieta (2002, pp. 1-36). En primer lugar, el rol que juega el lenguaje aún no es relevante en la tesis Dasein und Erkennen, como sí será a partir de su trabajo de habilitación (Apel 1975 [1963]). Esto conlleva un segundo elemento, que corresponde al lugar preponderante que posee la cuestión de la constitución del sentido, y no así el de la justificación de la validez. Es decir que aún no encuentran lugar aquí los elementos normativos de la comprensión. Ahora bien, a pesar de ello, como ya dijimos, el interés de fondo sigue siendo de tipo trascendental. Lo que le preocupa a Apel son las condiciones de posibilidad de la comprensión como radicalización y profundización existencial de la problemática de la constitución del conocimiento. Otro de los elementos que aparecen en la tesis son la concepción del sujeto y la consecuente superación del solipsismo metodológico y la autodiferenciación de la ciencia. En este último punto, puede observarse cómo la distinción entre explicar y comprender se deriva de los modos del «estar en» (Sein bei) y del «ser con» (Mit-sein) de Ser y Tiempo. En efecto, el primero correspondería a la experiencia o modo de ser existencial que está a la base del discurso objetivante y de conocimiento del mundo «externo». El segundo, en cambio, fundamentaría la ciencia de la comprensión como modo derivado de la relación del Dasein con los otros y consigo mismo. A modo de conclusión, entonces, podemos afirmar que ya en la primera obra de Apel la 
impronta heideggeriana es fundamental, que esa impronta no anula ni cancela otros caminos teóricos y críticos, que tal legado teórico o supuesto filosófico fundamental se sintetiza en la cuestión de la constitución del sentido, y que esta cuestión involucra la necesidad de plantear las condiciones de posibilidad a priori, es decir, presuponen una perspectiva trascendental.

\section{2 LENGUAJE Y APERTURA DEL MUNDO}

El segundo momento de la recepción crítica de Heidegger se circunscribe aproximadamente a los años 1955-1965. En este período, Apel elabora una serie de trabajos vinculados a la historia de las ideas. Desde su artículo, en el marco de la Begriffgeschichte sobre la historia del concepto de comprensión (Apel 1955) hasta su tesis de habilitación, publicada en 1963, Die Idee der Sprache in der Tradition des Humanismus von Dante bis Vico. La importancia de este trabajo excede el marco de la filosofía apeliana, puesto que se trata de una investigación que recupera y reconstruye una tradición que no entiende al lenguaje como cálculo o instrumento del pensamiento, sino que, siguiendo la tradición humboldtiana, lo comprende en cierto sentido como constituyente de la realidad ${ }^{5}$. En ese espíritu también están escritos los primeros textos de Apel reunidos en la primera parte del primer tomo de La transformación de la filosofía: «Lenguaje y apertura del mundo» (Sprache und Welterschliessung). El primero de esos textos, escrito hacia 1955, aborda las «dos fases» de evolución de la fenomenología y su impacto en la concepción de la poesía (Dichtung) y el arte en general. Se trata de un texto claramente influenciado por Heidegger que propone una «interpretación dialéctica» del desarrollo de la filosofía de su época, y es uno de los pocos textos en los que Apel se ocupa de estética, anticipándose incluso en algunos puntos a la hermenéutica de Gadamer (Verdad y Método se publica en 1960). El primer momento o tesis estaría representado por la tradición de las ciencias del espíritu y su antítesis, el naturalismo o positivismo. Según Apel, ambos movimientos coinciden en una «reducción óntica», esto es, en una reducción del mundo o de la realidad, ya sea a la naturaleza, como en el caso del positivismo, o al «espíritu», como es el caso de la hermenéutica. El segundo momento corresponde a la fenomenología de Husserl. Según Apel, el platonismo del significado de Husserl le permite a este enfrentarse a la reducción psicologista de los significados a procesos psicofísicos, cuestión que le permite además luchar contra el relativismo. Sin embargo, la fenomenología tenía que enfrentarse con el problema de la «historicidad del sentido» y del «aspecto creador del lenguaje» (Apel 1985 I, p. 85). Precisamente esta dificultad se encuentra en la obra de arte y en la literaria,

5 Un análisis detallado de la tesis de habilitación de Apel lo realiza Mendieta (2002, pp. $37-72)$. 
pues no puede pensarse (como lo hace Ingarden, según la interpretación de Apel) que la obra sea una esencia o entidad ideal autónoma e inmutable, sino que «el hombre tiene que buscar su [de la obra] propia unidad e identidad existenciales en la co-realización creadora o recreadora de la obra...» (Apel 1985 I, p. 88). El tercer momento o síntesis dialéctica se lograría, como era de esperar, con Heidegger. En este caso, señala Apel, «... el sentido del ser, adquiere la forma de una estructura de significación antes que nada en y por el lenguaje» (Apel 1985 I, p. 92), y luego agrega: «Siempre que el hombre acceda desde sus preferencias vitales a la comprensión de un ente en su esencia, el ser ya se ha instalado en la casa de un lenguaje» (Apel 1985 I, p. 92). Hasta este momento Apel se ocupa del aporte positivo de Heidegger, esto es, precisamente, del giro hermenéutico de la fenomenología husserliana. A continuación, se describe el aspecto crítico y las limitaciones que encuentra en la filosofía de Heidegger.

\section{I.3 EL PROBLEMA DE LA JUSTIFICACIÓN DE LA VALIDEZ}

En el Prólogo a La transformación de la filosofía, el propio Apel da cuenta de su giro, del que tal obra es testimonio. En efecto, allí el filósofo explica que el primer tomo de la misma está escrito todavía bajo la «fascinación que produce el acontecer de sentido (Sinn-Ereignis), nunca manipulable, que acaece en la apertura lingüística del mundo» (Apel 1985 I, p. 7). Aún así, la segunda parte del primer volumen, «Hermenéutica y crítica del sentido», ya supone una instancia más crítica y, por ello, podría considerársela como parte de este tercer momento y no del anterior, como afirma $\operatorname{Crelier}^{6}$ (2010, p. 229). En cuanto al segundo volumen de La transformación de la filosofía, Apel señala que este está motivado directamente «por el intento de lograr una orientación normativa en la línea de la justificación trascendental del conocimiento válido, en su más amplio sentido» (Apel 1985 I, p. 7). Además, en una nota a pie de página aclara: «Los trabajos impresos en la primera parte del primer volumen que han incurrido en gran parte en la confusión o mezcla del problema de la apertura del sentido y el de la verdad...» (Apel 1985 I, p. 40 n. 67) (cursiva G. S.). De algún modo, aquí Apel se hace eco de la crítica a Heidegger llevada a cabo por Tugendhat en su tesis Der Warheitsbegriff bei Husserl und Heidegger. Por otra parte, el texto de Tugendhat coincide históricamente con la «retrac-

6 De hecho, el propio autor se refiere aquí a la «distancia crítica», la «reflexión filosófica sobre la validez» y sobre la «normatividad» al referirse al artículo con el que abre la segunda parte del primer volumen de la La transformación de la filosofía (Crelier 2010, p. 230). Pero estas son cuestiones que ya están referidas no a la constitución del sentido, sino, precisamente a la justificación de la validez. El verdadero cambio de un momento a otro, es decir, de la fascinación por la apertura de la verdad a la reflexión sobre la validez se produce en el pasaje de la primera a la segunda parte de La transformación de la filosofía, tal como lo reconoce el propio Apel (1985 I, p. 40 n. 67). 
tación» de Heidegger en Zur Sache des Denkens ${ }^{7}$, y Apel incluso sugiere que Heidegger podría haber sido influenciado por Tugendhat ${ }^{8}$. Este es el punto fundamental en la crítica de Apel a Heidegger y unos veinticinco años más tarde, Lafont retomará esta cuestión, dándole un nuevo impulso. La tesis en la que convergen Tugendhat, Apel y Lafont afirma que Heidegger confunde o equipara la cuestión de la justificación de la validez con la de la constitución del sentido. Esta equiparación tendría además dos tipos de consecuencias. Por un lado, desde el punto de vista epistemológico, la imposibilidad de la verdad y del aprendizaje a partir de la experiencia debido a la pertenencia de todo conocimiento a un ámbito previo de sentido. En segundo lugar, habría una consecuencia de tipo ético-política correspondiente a la imposibilidad de trascender el propio contexto mundo-vital ${ }^{9}$.

\section{I.4 NUEVAS PERSPECTIVAS}

A modo de hipótesis se propone aquí considerar como un cuarto momento de la crítica a Heidegger algunos textos escritos desde fines de 1980, principalmente recogidos en Auseinandersetzungen ${ }^{10}$. Es cierto que en esencia la perspectiva sobre la filosofía de Heidegger no se modifica respecto de los trabajos de la década del ' 60 , basta tomar como ejemplo la mirada retrospectiva sobre la relación Heidegger-Wittgenstein en dicha obra. Sin embargo, en esta última etapa se destaca principalmente la influencia de la obra de Lafont sobre Heidegger y también las nuevas discusiones en torno a la teoría del significado (Apel 1998, pp. 549 ss.). En un artículo publicado en 2001, Apel sostiene que «los representantes de la 'semántica realista' -por ejemplo, Donellan, Kripke y Putnam- han mostrado que la tesis heideggeriana concerniente a la unilateralidad de la dependencia de toda proposición verdadera y falsa a la apertura lingüística es insostenible» (Apel 2001, p. 464). Si bien esta tesis de algún modo

7 Con ello se alude a la afirmación de Heidegger en el mencionado texto de que «aletheia, «desocultamiento» pensado en tanto que Lichtung de lo presente, no es todavía verdad» (Lafont 1997, p. 148).

8 Esto desatará además una polémica con Otto Pöggeler (Lafont, 1997: p. 148 n. 6).

9 En relación con el segundo punto mencionado, en la Introducción a La transformación de la filosofía Apel alude por primera vez, hasta donde sabemos, a la relación de Heidegger con la política y su compromiso con el nazismo. En una breve nota señala: «En este punto -y por razones precisamente filosóficas - no podemos silenciar la curiosa coincidencia con que se produjeron el giro heideggeriano hacia el destino del ser como acontecer del sentido $y$ como legitimación del sentido, y el error político de 1933» (Apel 1985 I: p. 39 n. 60).

10 Nos referimos principalmente a «Wittgenstein und Heidegger. Kritische Wiederholung und Ergänzung eines Vergleichs» (Apel 1998, pp. 459-503) y sobre todo a «Sinnkonstitution und Geltungsrechtfertigung. Heidegger und das Problem der Transzendentalphilosophie» (Apel 1998, pp. 507-568). 
afirma con otros términos algo similar a lo que ya venía señalando Apel, el nuevo «marco teórico» abre también otros problemas. En efecto, el problema que se plantea aquí es que las teorías de la referencia directa, particularmente el caso de Lafont, cuestionan severamente el «idealismo lingüístico» de la hermenéutica filosófica, y como «solución» proponen una rehabilitación de cierto tipo de realismo ${ }^{11}$.

El matiz peculiar que encontramos en los trabajos de Apel, desde mediados de los años '80, en relación con la hermenéutica filosófica, es que si bien se sigue reconociendo la dimensión de apertura del mundo del lenguaje, hay un mayor grado de problematización o de conciencia respecto de la cuestión referida al intensionalismo de la hermenéutica. Este vocabulario no se encuentra en la recepción apeliana de la hermenéutica en los años '60, entre otras cosas, porque es un tópico de la filosofía analítica, más específicamente de Putnam y su crítica a las teorías «intensionalistas» del significado, de la cual se vale Lafont para realizar la reconstrucción crítica de la obra de Heidegger.

\section{Hermenéutica Filosófica \\ Vs. HermenÉutica Trascendental}

Todo el programa filosófico de Apel, su sistema, por decirlo así, puede ser comprendido como el de una filosofía trascendental, y como tal, a pesar de sus críticas, es heredera de la tradición kantiana. En La transformación de la filosofía, Apel afirma:

11 En «Regulative Ideen oder Wahrheits-Geschehen?», Apel afirma que es posible la corrección del sentido mediante la referencia, y que «una 'hermenéutica filosófica' no sólo tiene que ver con el sentido intensional inmanente del lenguaje, sino también siempre con las pretensiones de validez objetivas que posee toda expresión con sentido» (Apel 1998, 582). Aquí es entonces donde Apel se refiere a conceptos como los de «designadores rígidos»o «natural kinds» que limitarían o restringirían el «como» hermenéutico. En el mismo texto, afirma: «La comprensión fundamental de Heidegger para el giro lingüistico-hermenéutico de la fenomenología, sobre la estructura del «como»... encuentra su correctivo en la muy plausible circunstancia de utilizar nombres como designadores rigidos que relacionen en la referencia directa libre del «como» en la deixis (mediante de expresiones indexicales) lo dado designado... Lo dado no es, en esta medida, reconocido todavía «como algo» (Apel 1998, 582). Aquí la dificultad u objeción que se puede plantear, desde una perspectiva hermenéutica, es que «lo dado» en la deixis, incluso como «mero juicio de percepción», se encuentra ya determinado por el sentido abierto en un estado de interpretado. No se trata de que «la cosa» que afecta, por extraña que sea, se presente como una mera resistencia a la voluntad, sino que la cosa misma se presenta como. Cuestionar la universalidad del como hermenéutico, desde mi punto de vista, significaría poner en tela de juicio la hermenéutica misma. 
«Me parece que la situación de la filosofía trascendental en la era de la ciencia está marcada por el hecho de que la filosofía no puede reclamar en modo alguno un objeto, como su objeto (ni la conciencia, ni el lenguaje, ni la sociedad qua comunidad de comunicación). Pero, en cambio, puede y debe investigar virtualmente todos los objetos del conocimiento, tanto científico como precientífico, en virtud de su valor trascendental como condiciones de posibilidad y de validez del conocimiento...» (Apel 1985 I, p. 68 n.115).

Esta reafirmación de un programa trascendental o del carácter trascendental de la filosofía puede ser ejemplificada con dos momentos del desarrollo de la filosofía de la segunda mitad del siglo XX que tienen especial impacto en el pensamiento de Apel. El primero, como se muestra a lo largo del presente trabajo, obedece a la lectura de Apel de Verdad y método (1960), que continúa o profundiza la idea de verdad como acontecimiento en el sentido de Heidegger. El segundo, que no puede ser abordado aquí, corresponde a la filosofía de Rorty. En los dos contextos, aquel de la hermenéutica de mediados de los años 1960 y el del neopragmatismo, Apel abogó por una «retrascendentalización» de la filosofía, es decir, por un retorno crítico a Kant. De acuerdo con el diagnóstico de Apel, la hermenéutica filosófica, sobre todo con Heidegger, tuvo la posibilidad de seguir también este camino trascendental o cuasi-trascendental, pero en lugar de ello optó por una subordinación total de la cuestión de la validez a la cuestión de la constitución histórico-temporal del sentido, es decir, la subordinación del logos mismo de la ciencia y la filosofía a la «historia del ser» que simplemente «acaece». No obstante, la filosofía trascendental clásica, en el caso de Kant, donde «la pregunta por las condiciones de posibilidad de la validez objetiva del conocimiento es idéntica a la pregunta por la constitución trascendental del sentido de la objetividad (Objetktivität) u objetualidad» (Gegenständlichkeit) (Apel 1998, p. 537), tampoco ofrece, para Apel, una salida viable, dado que parte de la idea de una «conciencia en general» y no reconoce la mediación histórico-lingüística del sentido. Por ello, Apel pretende elaborar una alternativa que reconozca tanto la historicidad de la constitución del sentido («precomprensión») «como presupuesto para la verdad o falsedad de los juicios de conocimiento [como] la validez universal y atemporal de esos juicios» (Apel 1998: p. 537) ${ }^{12}$ (cursiva, G. S.).

A diferencia de la interpretación de la filosofía de Heidegger que, como hemos mostrado en el primer punto, atraviesa distintos momentos, el posicionamiento respecto de Gadamer podría decirse que no se ha modificado desde

12 Ya en La transformación de la filosofía, Apel afirmaba: «La filosofía trascendental, en el sentido kantiano, difícilmente pueda superarse mediante una filosofía del destino del ser; más bien podemos ampliarla o profundizarla en la línea de una hermenéutica trascendental» (Apel 1985 I, p. 41) 
sus primeros escritos ${ }^{13}$. En efecto, al recorrer los trabajos específicos de Apel sobre la hermenéutica filosófica ${ }^{14}$, creemos que se pueden señalar tres puntos fundamentales en disputa, presentes de una u otra manera en todos ellos. En primer lugar, la oposición de Apel al carácter meramente «ontológico-temporal» de la comprensión, y consecuentemente su defensa de la idea regulativa de un comprender mejor como presupuesto de la comprensión. En segundo lugar, en conexión con la crítica a Heidegger, su cuestionamiento de la verdad como acontecimiento y el problema de una concepción intensional de la referencia. En tercer lugar, el rechazo de Gadamer a la reflexión y la defensa apeliana de la reflexión trascendental, que converge en una autodiferenciación de grados de discursos de entendimiento empírico-falible y trascendental-infalible (pero autocorregible).

\section{II.1 MODELO ONTO-HISTÓRICO VERSUS MODELO DIALÓGICO}

La publicación de Verdad y Método tuvo en el desarrollo filosófico de Apel un lugar especial. Como el propio Apel señala, ya en su tesis de habilitación utilizó la expresión «hermenéutica trascendental» para referirse a una posición que luego encontraría similitud con la de «hermenéutica filosófica» utilizada por Gadamer (Apel 1975, n. 61a, n. 62.). Precisamente, hasta ese momento Apel entendía la «hermenéutica del Dasein» propuesta por Heidegger como una transformación necesaria y suficiente (notwendige und zureichende) de la filosofía trascendental de Kant y Husserl, que tenía en cuenta la historicidad y la lingüisticidad. Sin embargo, la lectura de Verdad y Método disuadió a Apel de esa convicción. El punto central del disenso consistía (y consiste aún), según Apel, en la imposibilidad de pensar en un «progreso de la comprensión guiado

13 La única variante que se podría mencionar aquí es la discusión respecto de la crítica de las ideologías. Como es sabido, este programa es «abandonado» (o asumido) por Habermas en la Teoría de la acción comunicativa. En el caso de Apel, si bien no hay un rechazo de tal programa, sí hay una autocrítica: «Jürgen Habermas y yo sostuvimos -tal como lo veo hoy, sin razón- que la «pretensión de universalidad» de la hermenéutica filosófica de Gadamer podía ponerse en cuestión desde enfoques propios de las ciencias sociales, como el psicoanálisis y la crítica de las ideologías» (Apel 2002, p. 150-151) (cursiva G. S.).

14 Referencias a Gadamer pueden encontrarse a lo largo de toda la obra de Apel. Sin embargo, podrían señalarse algunos trabajos esenciales. En La transformación de la filosofía, los textos principales son: la Introducción (Apel 1985 I, p. 21 ss.), «Cientística, hermenéutica y crítica de las ideologías» (Apel 1985 II, pp. 91-119), «Cientificismo o hermenéutica trascendental?» (Apel 1985 II, pp. 169-208). En Auseinandersetzungen (Apel 1998, pp. 569-607) se encuentra recogido el artículo fundamental «¿Ideas regulativas o acontecer de la verdad? Sobre el intento de Gadamer de responder a la pregunta por las condiciones de posibilidad del comprender válido» (Apel 2002, pp. 134-167). Por último, un trabajo muy reciente, inédito en su lengua original, pero disponible en italiano e inglés: «Hermenéutica del ser versus hermenéutica trascendental» (Apel 2008). 
normativamente», dado que Gadamer identificaría, siguiendo a Heidegger, las «condiciones de posibilidad del sentido» con las «condiciones de posibilidad de la validez intersubjetiva de la comprensión» (Apel 1998, p. 572). Con ello se reemplaza el problema kantiano de la validez intersubjetiva contrafáctica por la facticidad del sentido abierto en cada situación, y esto, conducido por una modificación del concepto de verdad en el sentido de la aletheia. De tal modo, según Apel, Gadamer llevaría a cabo una transformación de los conceptos hermenéuticos tradicionales en conceptos «ontológico-temporales» (Apel 1998, p. 572). Desde la perspectiva de Apel, entonces, convive en la filosofía de Gadamer una tensión entre la historicidad radical de la comprensión (como acontecimiento) y el modelo dialógico, proveniente de Platón y no presente en Heidegger, de la relación yo-tu en el entendimiento (Verständigung) sobre la cosa. Es decir, se trata de una tensión entre la relación asimétrica del intérprete respecto del interpretandum y la relación simétrica entre dos interlocutores en el marco de un discurso o diálogo argumentativo, aún cuando tales interlocutores pertenezcan a «mundos de vida» radicalmente diferentes. Es decir, por ejemplo el lector actual de Platón está afectado por la «historia efectiva» de la tradición que él mismo se propone interpretar, y aunque claramente la «situación hermenéutica» sea radicalmente diferente a la del propio Platón, como lo que conduce el diálogo virtual con el autor es la cosa misma (pregunta, asunto o problema) es, precisamente, ese diálogo virtual el que pone al intérprete en una posición de interlocutor virtual.

La diferencia entre el comprender diferente de Gadamer y el comprender mejor radica no tanto en una diferencia acerca del fenómeno fáctico del comprender (Verstehen), sino en el punto de partida mismo, esto es, en aquello por lo que se pregunta. En efecto, la pregunta de Apel no es por las condiciones de posibilidad del comprender sin más, sino por las condiciones de posibilidad del comprender válido. Pero como toda cuestión relativa a la validez, no se refiere ya puramente a una instancia fáctica, sino contra-fáctica, entonces el comprender mejor debe ser entendido como una idea regulativa ${ }^{15}$ anticipada contrafácticamente en el acto mismo de la comprensión, y ello no sólo respecto de otras interpretaciones disponibles sino incluso del propio autor. Esto se debe a que Apel, al igual que Gadamer, no entiende la comprensión como referida a la presunta intencionalidad de un autor sino a la cosa misma sobre la que se está hablando. A partir del ejemplo anterior, deberíamos decir entonces que

15 Si bien hay diferencias importantes entre el enfoque trascendental de Apel y el «científico-reconstructivo» de Habermas, puede consultarse de este último el libro Acción comunicativa y razón sin trascendencia, donde hay una reconstrucción histórico-filosófica de las ideas de la razón pura de Kant entendidas ahora, en la filosofía discursiva, como «presupuestos idealizantes» (Habermas 2003). 
comprender a Platón no significaría comprender la «intencionalidad» del autor, «lo que quiso decir», sino aquello sobre lo que se está hablando. No obstante, esto no significa que el sentido del texto sea agotable o pueda volverse perfectamente transparente, sino tan sólo que junto al postulado «heurístico» de la superioridad del interpretandum debe mantenerse, en tensión dialéctica, el postulado del comprender crítico reflexivo del intérprete (Apel 2002, p. 138) ${ }^{16}$.

\section{II.2 VERDAD Y REFERENCIA}

$\mathrm{La}$ «utilidad» que ofrece una teoría de la verdad como aletheia a la hermenéutica podría llevar a considerar las pretensiones de sentido como el objeto mismo de la hermenéutica, es decir, como independiente de las pretensiones de validez que en dicho se sentido se formulan. Sin embargo, ello conduciría a la hermenéutica hacia el objetivismo que las ciencias del espíritu en el sentido clásico presuponían, y esto es precisamente lo que Gadamer quiere corregir. En este punto coinciden Apel y Gadamer, pues ambos parten del paradigma del «entendimiento comunicativo» (kommunikativen Verständigung), donde, tal como señala la palabra alemana «Verständigung», la comprensión del sentido (Verstehen) de algo está íntimamente ligado con el comprender-se (entenderse) con otro acerca de ese «algo». Sin embargo, aquí nos encontramos con una dificultad al momento de explicar qué sea ese «algo» (la cosa). En efecto, si la posibilidad del acuerdo sobre la cosa depende de un «acuerdo previo» o precomprensión sobre la base del lenguaje común compartido, en términos de Apel, el sentido intersubjetivamente compartible, entonces el referente resulta ser un elemento necesariamente intralingüístico. Este es el núcleo de la crítica de Lafont al «idealismo lingüístico» de la hermenéutica filosófica (Lafont 2002). Sin embargo, sorpresivamente, Apel afirma que es posible la corrección del sentido mediante la referencia, y que «una hermenéutica filosófica no sólo tiene que ver con el sentido intensional inmanente del lenguaje, sino también siempre con las pretensiones de validez objetivas que posee toda expresión con sentido» (Apel 1998, p. 582). Apel, del mismo modo que Lafont, utiliza herramientas de la filosofía analítica tales como los conceptos de «designadores rígidos» (Kripke) o «natural kinds» (Putnam), que limitarían o restringirían el «como» hermenéutico. Sin duda este es el punto más problemático, y el que aún requiere de una investigación más detallada, que excede los límites reconstructivos de este artículo.

Uno de los problemas fundamentales es que, de acuerdo con la «experiencia hermenéutica», no habría «referencia objetiva» pura, sino automediación de la tradición, por ello sería imposible pensar en un conocimiento

16 En otros términos, podría decirse que la comprensión permanece en la tensión entre «caridad» (Davidson) y «sospecha» (Ricoeur). 
progresivo sobre la cosa, en cuanto objeto acabado, en el ámbito de las ciencias del espíritu. Es decir, se podría conceder hasta cierto punto la identidad del significado para objetos de tipo naturales como agua u oro (Putnam), sin embargo, difícilmente podría sostenerse lo mismo para términos tales como «justicia», «crueldad», «bien común», etc. Dicho de otra manera, lo que sea la cosa, viene inscripto dentro de una tradición, de una comunidad lingüística. La identidad del significado está constituida por la pertenencia de los conceptos a una comunidad lingüística. Apel reconoce esto, incluso concede a Gadamer que «la referencia objetiva de todas las ciencias del espíritu históricas no se determina mediante un objeto acabado que deba investigarse progresivamente, sino mediante el acontecer irreversible de la historia, que ellas mismas cooperan a constituir a través de la prosecución de la tradición» (Apel 2002, p. 149). Sin embargo, Apel aclara que no debe confundirse el «ideal de objetividad», que Gadamer denuncia, con la validez intersubjetiva (Apel 2002, p.149). Ahora bien, al menos en estos trabajos donde se discute con Gadamer, la respuesta de Apel no es satisfactoria, porque lo que está en juego no es la cuestión de la validez intersubjetiva sino la posibilidad o no de «echar por tierra» el holismo del significado o la determinación de la referencia (extralingüística) mediante el significado intensional del lenguaje $\mathrm{e}^{17}$.

\section{II.3 REFLEXIÓN Y DISCURSOS DE ENTENDIMIENTO (VERSTÄNDIGUNGSDISKURS)}

Tal vez el punto de discordancia más importante entre la hermenéutica filosófica de Gadamer y la hermenéutica trascendental de Apel radica en el lugar que tiene la reflexión trascendental. Es muy conocido el rechazo de Gadamer en Verdad y método y también la respuesta ortodoxa de la pragmática tras-

17 A propósito, el propio Apel plantea la siguiente pregunta: «¿se puede mantener todavía la pretensión de que la mediación a través del lenguaje es una condición no-trascendible de la posibilidad de nuestra identificación de algo en tanto que algo y de esta manera de nuestro conocimiento intersubjetivamente válido del mundo?» (Apel 2009, p. 150). Como se ha venido señalando, tal posibilidad de identificación «más allá del lenguaje» es, desde un punto de vista hermenéutico, imposible. Pero, al mismo tiempo, si no fuera posible la identificación del denotatum real y toda extensión de los términos estuviese determinada por su intensión, entonces, nuevamente nos encontraríamos con la paradoja de la inconmensurabilidad entre lenguajes. Según Apel, para «salir» de este problema la filosofía post-analítica ofrece dos alternativas. Por un lado, mediante una reducción del significado a la intencionalidad mental, tal como podría encontrarse en el segundo Searle, y fuera de esta tradición, en Brentano y Husserl. Por otro lado, las teorías que ponen el acento en la extensionalidad de los términos, como es el caso de la mencionada teoría de la referencia directa, Kripke y Putnam. Sin embargo, Apel considera que ambas perspectivas tienen sus limitaciones, y la alternativa superadora debería partir de la tridimensionalidad de la semiosis, considerando cada momento, a saber, intencionalidad, extensionalidad e intensionalidad como co-originarios y complementarios. 
cendental tanto de Apel como de Kuhlmann (2009, pp. 135-148). Sin embargo, consideramos, con Crelier, que una respuesta creativa al impasse entre ambas tradiciones consiste en pensar la reflexión, y la filosofía discursiva de Apel, en términos de «circularidad» según el modelo del círculo hermenéutico (Crelier 2010, pp. 219 ss. $)^{18}$.

Apel distingue dos tipos de discursos de entendimiento (Verständigungsdiskurs): el discurso del mundo de la vida o de las ciencias del espíritu y el discurso filosófico como el del propio Gadamer que, en cuanto filósofo, analiza el discurso del mundo de la vida y de las ciencias del espíritu como acontecimiento de sentido y de verdad (Apel 2002, p. 154). En efecto, en tanto y en cuanto se permanezca dentro del ámbito del discurso filosófico, que es el plano de la reflexión sobre la validez de todo discurso, las proposiciones enunciadas son, aunque no use este término, transhistóricas. Por ejemplo, la tesis del falibilismo (acerca de la falibilidad de toda teoría empírica) no puede ella misma ser falible, si es que no se quiere cometer (y permanecer en) una contradicción performativa. Para Apel, hay una relación estrecha entre el discurso reflexivo de la filosofía y los discursos hermenéuticos-empíricos (crítica de las ideologías, hermenéutica de la sospecha, la genealogía en el sentido de Nietzsche o Foucault, etc.), en la medida en que el discurso filosófico «rebasa la sujeción del comprender a la perspectiva» y permite nuevas «aperturas de

18 Crelier afirma «la imposibilidad de contar con un método o procedimiento infalible que explicite, desde un punto de vista distanciado y neutral, las condiciones de posibilidad de la experiencia o de la argumentación sin asumirlas» (Crelier 2010, p. 209). De esta manera, se distancia de una «línea dura» de la pragmática trascendental, representada por el propio Apel y por su discípulo Kuhlmann. En efecto, si no es posible un método de fundamentación última que se distancie de lo observado (intentio recta), como, según Crelier, sugiere la pragmática trascendental, entonces debemos asumir la inevitabilidad de la circularidad de la fundamentación (intentio obliqua). En otros términos, parafraseando a Heidegger, lo importante no es evitar la circularidad (algo de hecho imposible), sino mostrar la manera correcta de ingresar en ella. El procedimiento para la fundamentación última debe, entonces, estar guiado por «indicaciones realizativas» (performativas) que no señalan «desde afuera» sino que se encuentran involucradas performativamente en los presupuestos trascendentales. «Por ejemplo, dice Crelier, la exigencia pragmático-trascendental de utilizar el diálogo argumentativo como manera de llevar adelante una fundamentación última se presenta ella misma de manera dialógica» (Crelier 2010, p. 319). Según el autor, tales indicaciones son: la dialogicidad argumentativa, la auto-reflexión y la crítica del sentido. Los presupuestos últimos no pueden en sentido estricto ser demostrados, sino tan sólo pueden ser refutados aquellos que intenten negarlo. Por ejemplo, quien afirmara que no hay posibilidad de expresar enunciados con sentido, lo hace expresando un enunciado con sentido, por lo tanto se refuta a sí mismo. Pero, precisamente, para advertir esta inconsistencia es necesario un movimiento de reflexión, es decir, de volver y revisar la propia situación argumentativa: este es el procedimiento auto-reflexivo. Por último, la «crítica del sentido» consiste en mostrar en qué casos se traspasa los límites del sentido, los cuales funcionan como «el suelo» de la argumentación. 
sentido» (Apel 2002, p. 155). La prueba de tal afirmación la ofrece, según Apel, el propio descubrimiento que realiza la ética discursiva de normas universalmente válidas. Estas «verdades prácticas» son alcanzadas por medio de una reflexión de la propia razón entendida como discurso argumentativo y no por caminos empíricos, pero tal descubrimiento (por ejemplo de la igualdad y corresponsabilidad de todo interlocutor en un discurso) tiene un valor en cuanto crítica social o cultural de las sociedades empíricamente existentes. Es decir, la reflexión trascendental llevada a cabo en y por el discurso filosófico abre una instancia crítico-normativa de los discursos empíricos. Sin embargo, tal reflexión filosófica no es externa a la facticidad (situación histórica, «precomprensión»), ni es meramente formal, sino que se trata de un «volver sobre» la situación argumentativa actual fácticamente irrebasable.

\section{A MODO DE CONCLUSIÓN}

En el presente trabajo hemos examinado el lugar que ocupa la hermenéutica filosófica en la filosofía de Apel. Dicho lugar no se reduce simplemente a un momento histórico, es decir, a algo así como una etapa «primitiva» en el desarrollo de la pragmática trascendental. Por el contrario, el propio Apel se ha referido a hermenéutica trascendental y pragmática trascendental como elementos complementarios de una semiótica trascendental en cuanto filosofía primera. Si esto es así, queda planteada entonces la pregunta por las diferencias entre hermenéutica y pragmática. Siguiendo a Burckhart, podemos señalar entonces que la hermenéutica (trascendentalmente transformada) a de ocuparse de las condiciones de posibilidad y validez de la constitución del sentido, mientras que la pragmática (trascendental) de la esfera formal de justificación de la validez. A partir de este esquema o esta delimitación (que es simplemente analítica) puede reclamarse para la hermenéutica trascendental una serie de problemas propios, un programa de investigación autónomo, aunque no aislado de la pragmática trascendental. El objetivo principal de este trabajo consistía en, precisamente, mostrar ese campo y los problemas planteados por él, abstrayéndose metodológicamente de las presuntas soluciones o respuestas a tales problemas. Para ello, dividimos la tarea en dos partes. En la primera se propuso un esquema de interpretación acerca de la recepción de Heidegger en la filosofía apeliana. A diferencia de otras interpretaciones, sostuvimos que dicha recepción puede ser divida en cuatro momentos, donde es precisamente el cuarto y último el que se presenta como novedoso en cuanto a los estudios sobre Apel se refiere. En este momento, se destacan los estudios de Apel sobre las teorías del significado, las críticas a Kripke y Putnam, y el rol que ocupa la interpretación de Lafont sobre Heidegger. Como se intentó mostrar, la crítica de Lafont no sustituye la mirada apeliana sobre Heidegger, que proviene de 
sus primeros escritos y de la lectura de Tugendhat, pero sí ubica el problema de la constitución del sentido en otro marco conceptual.

Ahora bien, la reconstrucción histórica no persigue un interés meramente histórico, sino un interés problemático y sistemático. Precisamente, la configuración del campo temático-problemático de la hermenéutica trascendental se da de manera completa cuando interviene Gadamer en la discusión. En el trabajo sostenemos la hipótesis, por supuesto falible, de que tal campo se organiza en torno a tres cuestiones. En primer lugar, a la cuestión del «enfrentamiento» de modelos de comprensión hermenéutica, presentes de manera tensional en el propio Gadamer. Esto es, el modelo «asimétrico» de la pertenencia a la tradición y el modelo «simétrico» de igualdad dialógica (de dos interlocutores en situación argumentativa). En segundo lugar, en relación con el problema de la verdad y la referencia extralingüística. Lo que se analiza en este punto, es la acusación que recibe la hermenéutica de «idealismo lingüístico», y por lo tanto de relativismo. En tercer lugar, en relación con la autodeterminación de la propia razón o los grados de discursividad. En este último punto, entonces, la filosofía trascendental de Apel no sólo se distingue de la hermenéutica filosófica, sino incluso de la pragmática formal propuesta por Habermas, ya que apuesta a una fundamentación última de nuestro conocimiento.

\section{REFERENCIAS BIBLIOGRÁFICAS}

APEL, K.-O.1975: Die Idee der Sprache in der Tradition des Humanismus von Dante bis Vico. $2^{\mathrm{a}}$ ed. Bonn: Bouvier

APEL, K.-O. 1985: La transformación de la filosofía. 2 Tomos. Madrid: Taurus

APEL, K.-O. 1989: «El desafío de una crítica total de la razón y el programa de una teoría de los tipos de racionalidad», en Anales de la Cátedra Fracisco Suárez, $\mathrm{n}^{\mathrm{o}} 29,63-95$

APEL, K.-O. 1998:Auseinandersetzungen in Erprobungdes transzendentalpragmatischen Ansatzes. Frankfurt: Suhrkamp

APEL, K.-O. 2001 a: «Pragmatism as Sense-Critical Realism Based on a Regulative Idea of Truth: In Defense of a Peircean Theory of Reality and Truth», en Transactions of the Charles S. Peirce Society, XXXVII, 4, 443-474

APEL, K.-O. 2001 b: The Response of Discourse Ethics to the Moral Challenge of the Human Situation as Such and Specially Today. Leuven: Peeters

APEL, K.-O. 2002: Semiótica transcendental y filosofía primera. Madrid: Síntesis

APEL, K.-O. 2008: "The hermeneutics of Being (Heidegger, Gadamer) versus transcendental hermeneutics or transcendental pragmatics (Apel)», en The Routledge Companion to Twenty Century Philosophy, London and New York: Routledge, 736-783

APEL, K.-O. 2009: Semiótica filosófica. Buenos Aires: Prometeo libros

APEL, K.-O. 2011: Paradigmen der Ersten Philosophie. Zur reflexiven transzendentalpragmatischen Rekonstruktion der Philosophiegeschichte, Frankfurt: Suhrkamp 
El impacto de Heidegger y Gadamer en la hermenéutica...

BERTORELLO, A. 2007: «La reflexividad del logos hermenéutico. El problema de la universalidad en la filosofía de Sein und Zeit», en Michelini, D. et al. (eds.). Ética del discurso. Recepción y críticas desde América Latina. Río Cuarto: Ediciones del ICALA

BOCCIGNONE, M. 2004: «Esserci e cognoscere. Riflesione trascendentale e anilitica esistenziale nel primo Apel», en Fenomenologia e societa, XXVII, n³, 103-120

BÖHLER, D. 1981: «Philosophische Hermeneutik und hermeneutische Methode«, en Fuhrmann, M. et al. (eds.), Text und Applikation, München: Wilhelm Fink Verlag, 483-511

BURCKHART, H. 2000: «Nichthintergehbarkeit und Unverzichtbarkeit einer diskursethischen Begründung von Moral«, en Burckhart, H, Reich, K. (eds.), Begründung von Moral: Diskursethik versus Konstruktivismus, Würzburg: Königshausen und Neumann

CENTENO, A. 2011: La antropología del conocimiento en la filosofía de Karl-Otto Apel. Universidad de Granada (tesis doctoral inédita).

CARMAN, T. 2002: «Was Heidegger a Linguistic Idealist?», Inquiry 45, 206-215

CONILL, J. 2010: Ética hermenéutica. Madrid: Tecnos

CRELIER, A. 2010: De los argumentos trascendentales a la hermenéutica trascendental. La Plata: Edulp

FUNKE, G. 1959: «Fenomenología trascendental y «filosofía primera». Observaciones al `Ensayo sobre una historia crítica de las ideas` de Husserl», Diánoia 5 (5), 150-194

GRONDIN, J. 1999: Introducción a la filosofía hermenéutica. Barcelona: Herder GRONDIN, J. 2003: Introducción a Gadamer. Barcelona: Herder

HABERMAS, J. 2002: Verdad y justificación. Madrid: Trotta

HEIDEGGER, M. 1963: Sein und Zeit, Tübingen: Max Niemeyer

KOSLOWSKI, P. 1997: «A Philosophy of Historical School: Erich Rothacker's Theory of Geisteswissenschaften», en Methodology of Social Sciences, Ethics, Economics and in the Newer Historical School: from Max Weber and Rickert to Sombart and Rothacker, Berlin-Heidelberg: Springer

KETTNER, M. 2011: «Hat Apel in den Sechzigerjahren mit Apel gegen Apel gedacht?«, disponible en http://www.filosofiefabrik.de/ (última consulta: 02/09/2013)

KUHLMANN, W. 2009: Unhintergehbarkeit. Studien zur Transzendentalpragmatik. Würzburg: Königshausen \& Neumann

LAFONT, C. 1997: Lenguaje y apertura de mundo: El giro lingüístico de la hermenéutica de Heidegger. Madrid: Alianza

LAFONT, C. 2002: The Linguistic Turn in Hermeneutic Philosophy, MIT Press

MENDIETA, E. 2002: The Adventures of Transcendental Philosophy: Karl-Otto Apel's Semiotics and Discourse Ethics. Rowman \& Littlefield

PETRAS, W. 2011: «Sinnkonstitution und Geltungsrechtfertigung. Zum Verhältnis von transzendentaler Hermeneutik un Transzendentalpragmatik in Kontexten einer zureichenden Vernunftbegründung«, Universität zu Köln (tesis doctoral inédita)

SALERNO, G. 2008: «Lenguaje, verdad e intersubjetividad: El influjo de Heidegger en la filosofía de Apel», en Revista de Filosofía, Aurora, Curitiba 20 (17), 413-431

SCHATZKI, T. 2005: «Early Heidegger on Sociality», en Dreyfus, H. y Wrathall, M. 
(eds.), A Companion to Heidegger, Blackwell Publishing

SCIVOLETTO, G. 2011 a: «Signo y lenguaje. Acerca de la interpretación de Apel y Lafont de Ser y Tiempo», en Tópicos. Revista de filosofía (Santa Fe), n² 22, 231-248

SCIVOLETTO, G. 2011 b: «Comprender y valorar: Acerca de la necesidad, dificultad y posibilidad de «evaluar críticamente» un mundo de vida extraño», en Michelini, D. et al. (eds.). Ética del discurso. Desafíos de la interculturalidad y la religión en un mundo global. Río Cuarto: Ediciones del ICALA, 70-82

SCIVOLETTO, G 2012 a: «La fundación práctica de la teoría desde un punto de vista hermenéutico-trascendental», en Práxis filosófica, Universidad del Valle (CaliColombia), $n^{\circ} 34,149-163$

SCIVOLETTO, G. 2012 b: «Repensar el humanismo y la racionalidad: Aportes desde la filosofía de Karl-Otto Apel», en Hermenéutica Intercultural, Santiago de Chile, $n^{\circ} 20-21,105-123$

SCIVOLETTO, G. 2012 c: «Democracia y pluralidad de las formas de vida», en Michelini, D. et al. (ed.). Ética del Discurso. Su significación para la filosofía práctica y el diálogo intercultural, Río Cuarto: Ediciones del ICALA, 106-114

TUGENDHAT, E. 1967: Die Wahrheitsbegriff bei Husserl und Heidegger, Berlin: de Gruyter.

Gonzalo Martín Scivoletto. Conicet.

Lineas de investigación:

Pragmática Trascendental, Hermenéutica, Ética del discurso, Filosofía del Lenguaje

Publicaciones recientes:

SCIVOLETTO, G. "La fundación práctica de la teoría desde un punto de vista hermenéuticotrascendental”. En: Práxis filosófica, Universidad del Valle (Cali-Colombia), nº 34, enero-julio 2012, 149-163 (ISSN: 0120-4688)

SCIVOLETTO, G. "Repensar el humanismo y la racionalidad: Aportes desde la filosofía de Karl-Otto Apel”. En: Hermenéutica Intercultural, Santiago de Chile, Universidad Católica Silva Henríquez, nº 20-21, 2012, (ISSN 0718-4980)

Dirección electrónica:

scivolettog@gmail.com

Dirección postal:

Sargento Cabral 327, Departamento 7

Godoy Cruz, Mendoza, Argentina

CP 5501 